Proyecciones Journal of Mathematics

Vol. 33, $\mathrm{N}^{\circ}$ 2, pp. 147-155, June 2014.

Universidad Católica del Norte

Antofagasta - Chile

\title{
The forcing connected detour number of a graph
}

\author{
A. P. Santhakumaran \\ Hindustan University, India \\ S. Athisayanathan \\ St. Xavier's College, India \\ Received : August 2013. Accepted : December 2013
}

\begin{abstract}
For two vertices $u$ and $v$ in a graph $G=(V, E)$, the detour distance $D(u, v)$ is the length of a longest $u-v$ path in $G$. A u-v path of length $D(u, v)$ is called a $u-v$ detour. A set $S \subseteq V$ is called a detour set of $G$ if every vertex in $G$ lies on a detour joining a pair of vertices of $S$. The detour number $\operatorname{dn}(G)$ of $G$ is the minimum order of its detour sets and any detour set of order $\operatorname{dn}(G)$ is a detour basis of $G$. A set $S \subseteq V$ is called a connected detour set of $G$ if $S$ is detour set of $G$ and the subgraph $G[S]$ induced by $S$ is connected. The connected detour number $c d n(G)$ of $G$ is the minimum order of its connected detour sets and any connected detour set of order $c d n(G)$ is called a connected detour basis of $G$. A subset $T$ of a connected detour basis $S$ is called a forcing subset for $S$ if $S$ is the unique connected detour basis containing T. A forcing subset for $S$ of minimum cardinality is a minimum forcing subset of $S$. The forcing connected detour number of $S$, denoted by $f c d n(S)$, is the cardinality of a minimum forcing subset for $S$. The forcing connected detour number of $G$, denoted by $f c d n(G)$, is $f c d n(G)=\min \{f c d n(S)\}$, where the minimum is taken over all connected detour bases $S$ in $G$. The forcing connected detour numbers of certain standard graphs are obtained. It is shown that for each pair $a, b$ of integers with $0 \leq a<b$ and $b \geq 3$, there is a connected graph $G$ with $f c d n(G)=a$ and $c d n(G)=b$.
\end{abstract}

Key Words : Detour, connected detour set, connected detour basis, connected detour number, forcing connected detour number. AMS

Subject Classification : 05C12. 


\section{Introduction}

By a graph $G=(V, E)$ we mean a finite undirected graph without loops or multiple edges. The order and size of $G$ are denoted by $p$ and $q$ respectively. We consider connected graphs with at least two vertices. For basic definitions and terminologies we refer to $[1,5]$. For vertices $u$ and $v$ in a connected graph $G$, the detour distance $D(u, v)$ is the length of a longest $u-v$ path in $G$. A $u-v$ path of length $D(u, v)$ is called a $u-v$ detour. It is known that the detour distance is a metric on the vertex set $V$. Detour distance and detour center of a graph were studied in $[2,4]$.

A vertex $x$ is said to lie on a $u-v$ detour $P$ if $x$ is a vertex of $P$ including the vertices $u$ and $v$. A set $S \subseteq V$ is called a detour set if every vertex $v$ in $G$ lies on a detour joining a pair of vertices of $S$. The detour number $d n(G)$ of $G$ is the minimum order of a detour set and any detour set of order $d n(G)$ is called a detour basis of $G$. A vertex $v$ that belongs to every detour basis of $G$ is a detour vertex in $G$. If $G$ has a unique detour basis $S$, then every vertex in $S$ is a detour vertex in $G$. These concepts were studied in [3]. A set $S \subseteq V$ is called a connected detour set of $G$ if $S$ is a detour set of $G$ and the subgraph $G[S]$ induced by $S$ is connected. The connected detour number $c d n(G)$ of $G$ is the minimum order of its connected detour sets and any connected detour set of order $c d n(G)$ is called a connected detour basis of $G$. A vertex $v$ in $G$ is a connected detour vertex if $v$ belongs to every connected detour basis of $G$. If $G$ has a unique connected detour basis $S$, then every vertex in $S$ a connected detour vertex of $G$. The connected detour number of a graph was introduced and studied in [6].

For the graph $G$ given in Figure 1.1, the sets $S_{1}=\left\{v_{1}, v_{3}\right\}, S_{2}=\left\{v_{1}\right.$, $\left.v_{5}\right\}$ and $S_{3}=\left\{v_{1}, v_{4}\right\}$ are the three detour bases of $G$ so that $d n(G)=2$. It is clear that no two element subset of $V$ is a connected detour set of $G$. However the set $S_{4}=\left\{v_{1}, v_{2}, v_{3}\right\}$ is a connected detour basis of $G$ so that $c d n(G)=3$. Also the set $S_{5}=\left\{v_{1}, v_{2}, v_{5}\right\}$ is another connected detour basis of $G$. Thus there can be more than one connected detour basis for a graph $G$. 


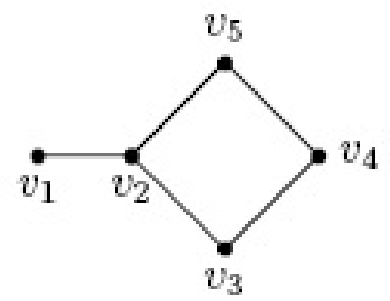

Figure 1.1: $G$

Graphs are often used to model network of real life problems and some definite part is always present in a minimum possible spanning set in a particular problem. For each connected detour basis $S$ in a connected graph $G$, there is always some subset $T$ of $S$ that uniquely determines $S$ as the connected detour basis containing $T$. Such subsets are called forcing subsets for $S$, and in this paper we briefly describe the properties satisfied by these sets in a graph.

The following theorem is used in the sequel.

Theorem 1.1. [6] All the end vertices and all the cut vertices of a connected graph $G$ belong to every connected detour set of $G$.

Throughout this paper $G$ denotes a connected graph with at least two vertices.

\section{The Forcing Connected Detour Number}

Definition 2.1. Let $G$ be a connected graph and $S$ a connected detour basis of $G$. A subset $T \subseteq S$ is called a forcing subset for $S$ if $S$ is the unique connected detour basis containing $T$. A forcing subset for $S$ of minimum cardinality is a minimum forcing subset of $S$. The forcing connected detour number of $S$, denoted by $f c d n(S)$, is the cardinality of a minimum forcing subset for $S$. The forcing connected detour number of $G$, denoted by $f c d n(G)$, is $f c d n(G)=\min \{f c d n(S)\}$, where the minimum is taken over all connected detour bases $S$ in $G$. 
Example 2.2. For the graph $G$ given in Figure 2.1, $S_{1}=\{u, s, w, t, v\}$ is the unique connected detour basis of $G$ so that $f c d n(G)=0$ and for the graph $G$ given in Figure 1.1, $S_{2}=\left\{v_{1}, v_{2}, v_{3}\right\}$ and $S_{3}=\left\{v_{1}, v_{2}, v_{5}\right\}$ are the only connected detour bases of $G$ so that $f c d n(G)=1$.

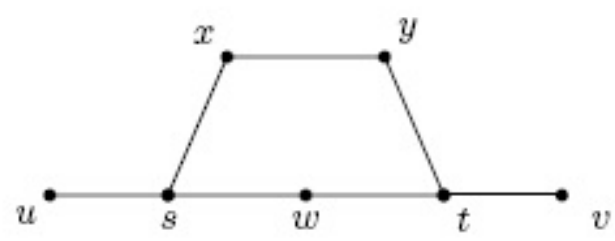

Figure 2.1: $G$

The next theorem follows immediately from the definitions of connected detour number and forcing connected detour number of a connected graph $G$.

Theorem 2.3. For every connected graph $G, 0 \leq f c d n(G) \leq c d n(G)$.

Remark 2.4. The lower bound in Theorem 2.3 is sharp. For the graph $G$ given in Figure 2.2, $f c d n(G)=0$. Also, all the inequalities in Theorem 2.3 can be strict. For the graph $G$ given in Figure 1.1, $c d n(G)=3$ and $f c d n(G)=1$. Thus $0<f c d n(G)<c d n(G)$.

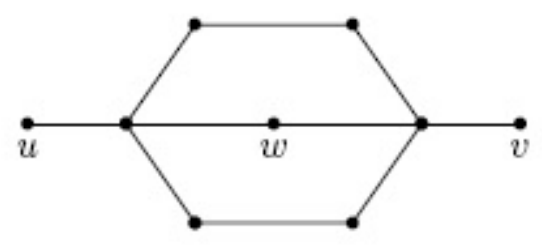

Figure 2.2: $G$ 
The following theorem is an easy consequence of the definition of forcing connected detour number of a graph.

Theorem 2.5. Let $G$ be a connected graph. Then

a) $f c d n(G)=0$ if and only if $G$ has a unique connected detour basis,

b) $f c d n(G)=1$ if and only if $G$ has at least two connected detour bases, one of which is a unique connected detour basis containing one of its elements, and

c) $f c d n(G)=c d n(G)$ if and only if no connected detour basis of $G$ is the unique connected detour basis containing any of its proper subsets.

Theorem 2.6. Let $G$ be a connected graph and $W$ be the set of all connected detour vertices of $G$. Then $f c d n(G) \leq c d n(G)-|W|$.

Proof. Let $S$ be a connected detour basis $S$ of $G$. Then $c d n(G)=|S|$, $W \subseteq S$ and $S$ is the unique connected detour basis containing $S-W$. Thus $f c d n(S) \leq|S-W|=|S|-|W|=c d n(G)-|W|$ and the result follows.

Corollary 2.7. If $G$ is a connected graph with $k$ end-vertices and $l$ cutvertices, then $f c d n(G) \leq c d n(G)-k-l$.

Proof. This follows from Theorems 1.1 and 2.6.

Remark 2.8. The bound in Theorem 2.6 is sharp. For the graph $G$ given in Figure 1.1, $\operatorname{cdn}(G)=3,|W|=2$ and $f c d n(G)=1$ as in Remark 2.4. Also, the inequality in Theorem 2.6 can be strict. For the graph $G$ of Figure 2.3, the sets $S_{1}=\left\{v_{1}, v_{2}, v_{3}, v_{4}, v_{7}, v_{8}\right\}$, and $S_{2}=\left\{v_{1}, v_{2}, v_{5}, v_{6}, v_{7}, v_{8}\right\}$ are the two connected detour bases of $G$ and $W=\left\{v_{1}, v_{2}, v_{7}, v_{8}\right\}$ so that $c d n(G)=6,|W|=4$ and $f c d n(G)=1$. Thus $f c d n(G)<c d n(G)-|W|$ - Moreover, the bound in Corollary 2.7 is also sharp. For the graph $G$ given in Figure 1.1, $\operatorname{cdn}(G)=3, k=1, l=1$ and $f c d n(G)=1$. Also, the inequality in Corollary 2.7 can be strict. For the graph $G$ of Figure 2.3, $c d n(G)=6, k=2, l=2$ and $f c d n(G)=1$. Thus $f c d n(G)<c d n(G)-k-l$. 


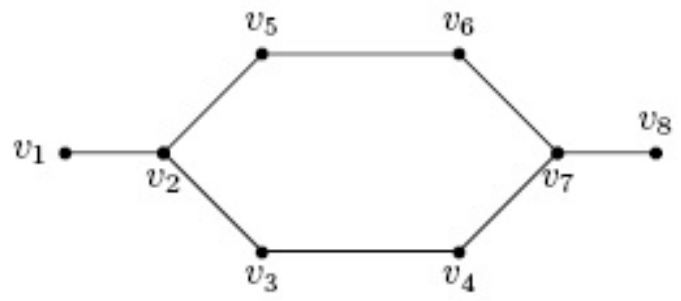

Figure 2.3: $G$

In the following theorems we proceed to find the forcing numbers of certain graphs $G$.

Theorem 2.9. Let $G$ be the complete graph $K_{p}(p \geq 2)$ or the cycle $C_{p}$ or the complete bipartite graph $K_{m, n}(2 \leq m \leq n)$. Then a set $S$ of vertices is a connected detour basis if and only if $S$ consists of any two adjacent vertices of $G$. Furthermore, $c d n(G)=2$ for each of these graphs.

Proof. If $G$ is the complete graph $K_{p}(p \geq 2)$ or the cycle $C_{p}$, then it is clear that any set of two adjacent vertices is a connected detour basis of $G$. Let $G$ be the complete bipartite graph $K_{m, n}(2 \leq m \leq n)$. Let $X$ and $Y$ be the bipartite sets of $K_{m, n}(2 \leq m \leq n)$ with $X=$ $\left\{x_{1}, x_{2}, \ldots, x_{m}\right\}$. Let $u \in X$ and $v \in Y$. It is clear that $D(u, v)=$ $2 m-1$. Let $y \in Y-\{v\}$. Then the vertex $y$ lies on a $u-v$ detour $P: u=x_{1}, y, x_{2}, y_{1}, x_{3}, y_{2}, \ldots, x_{m-1}, y_{m-2}, x_{m}, v$, where $y_{1}, y_{2}, \ldots, y_{m-2} \in$ $Y-\{v, y\}$. Thus the set $\{u, v\}$ is a connected detour basis of $K_{m, n}$.

Now, let $S$ be a connected detour basis of $G$. Let $S^{\prime}$ be any set consisting of two adjacent vertices of $G$. Then as in the first part of this theorem $S^{\prime}$ is a connected detour basis of $G$. Hence $|S|=\left|S^{\prime}\right|=2$ and it follows that the two vertices of $S$ are adjacent. The converse is obvious.

Theorem 2.10. a) If $G$ is the complete graph $K_{p}(p \geq 3)$ or the the cycle $C_{p}$ or the complete bipartite graph $K_{m, n}(m, n \geq 2)$, then $\operatorname{cdn}(G)=$ $f c d n(G)=2$.

b) If $G$ is a tree of order $p \geq 2$, then $c d n(G)=p$ and $f c d n(G)=0$. 
Proof. a) By Theorem 2.9, a set $S$ of vertices is a connected detour basis of $G$ if and only if $S$ consists of two adjacent vertices of $G$. For each vertex $v$ in $G$ there are at least two vertices adjacent with $v$. Thus the vertex $v$ belongs to more than one connected detour basis of $G$. Hence it follows that no set consisting of a single vertex is a forcing subset for any connected detour basis of $G$. Thus $f c d n(G)=2$. Also, by Theorem 2.9, $\operatorname{cdn}(G)=2$ and the result follows.

b) By Theorem 1.1, $c d n(G)=p$. The set of all vertices of a tree is the unique connected detour basis so that $f c d n(G)=0$ by Theorem 2.5(a).

Theorem 2.11. Let $G$ be a connected graph with cut-vertices and $S$ a connected detour set of $G$. Then for any cut-vertex $v$ of $G$, every component of $G-v$ contains an element of $S$.

Proof. Let $v$ be a cut-vertex of $G$ such that one of the components, say $C$ of $G-v$ contains no vertex of $S$. Let $u \in V(C)$. Since $S$ is a connected detour set of $G$, there exist vertices $x, y \in S$ such that the vertex $u$ lies on some $x-y$ detour $P: x=u_{0}, u_{1}, \ldots, u, \ldots, u_{t}=y$ in $G$. Let $P_{1}$ be the $x-u$ subpath of $P$ and $P_{2}$ be the $u-y$ subpath of $P$. Since $v$ is a cut-vertex of $G$ both $P_{1}$ and $P_{2}$ contain $v$ so that $P$ is not a detour, which is a contradiction. Thus every component of $G-v$ contains an element of $S$.

Theorem 2.12. Let $G=\left(K_{n_{1}} \cup K_{n_{2}} \cup \ldots \cup K_{n_{r}} \cup k K_{1}\right)+v$ be a block graph of order $p \geq 4$ such that $r \geq 1$, each $n_{i} \geq 2$ and $n_{1}+n_{2}+\ldots+n_{r}+k=p-1$. Then $\operatorname{cdn}(G)=r+k+1$.

Proof. Let $u_{1}, u_{2}, \ldots, u_{k}$ be the end-vertices of $G$. Let $S$ be any connected detour set of $G$. Then by Theorem 1.1, $v \in S$ and $u_{i} \in S(1 \leq$ $i \leq k)$. Also by Theorem 2.11, $S$ contains a vertex from each component $K_{n_{i}}(1 \leq i \leq r)$. Now, choose exactly one vertex $v_{i}$ from each $K_{n_{i}}$ such that $v_{i} \in S$. Then $|S| \geq r+k+1$. Let $T=\left\{v, v_{1}, v_{2}, \ldots, v_{r}, u_{1}, u_{2}, \ldots\right.$, $\left.u_{k}\right\}$. Since every vertex of $G$ lies on a detour joining a pair of vertices of $T$, it follows that $T$ is a detour basis of $G$. Also, since $G[T]$ is connected, $c d n(G)=r+k+1$.

Now, in view of Theorem 2.3, we have the following realization result.

Theorem 2.13. For each pair $a, b$ of integers with $0 \leq a<b$ and $b \geq 3$, there is a connected graph $G$ with $f c d n(G)=a$ and $c d n(G)=b$. 
Proof. Case 1: $a=0$. For each $b \geq 3$, let $G$ be a tree with $b$ vertices. Then $f c d n(G)=0$ and $c d n(G)=b$ by Theorem 2.10(b).

Case 2: $a \geq 1$. For each integer $i$ with $1 \leq i \leq a$, let $F_{i}$ be a copy of the complete graph $K_{2}$, where $V\left(F_{i}\right)=\left\{u_{i}, v_{i}\right\}$ and let $H=K_{1, b-a-1}$ be the star whose vertex set is $W=\left\{z_{1}, z_{2}, \ldots, z_{b-a-1}, v\right\}$. Then the graph $G$ is obtained by joining the central vertex $v$ of $H$ to the vertices of $F_{1}, F_{2}, \ldots, F_{a}$. The graph $G$ is connected and is shown in Figure 2.4.

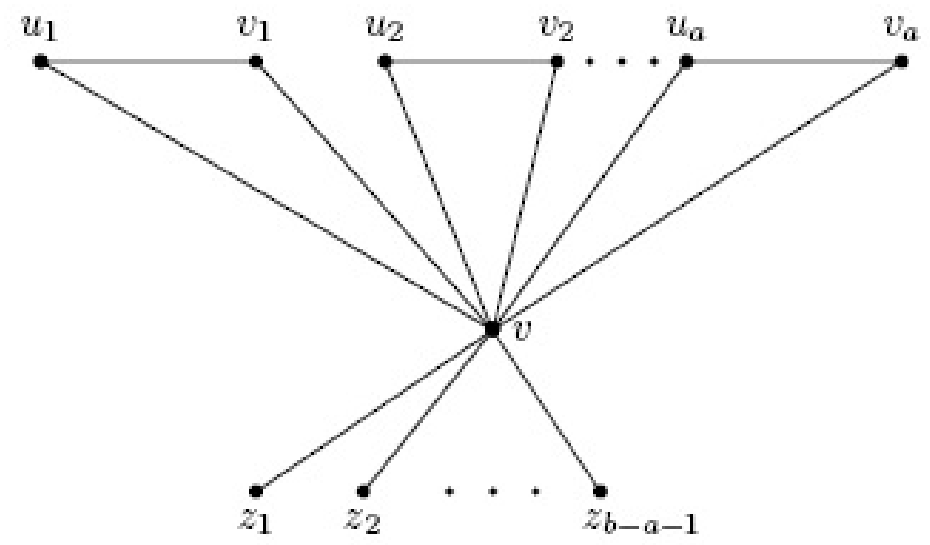

Figure 2.4: $G$

By Theorem 2.12, $c d n(G)=b$. Now, we show that $f d n(G)=a$. It is clear that $W$ is the set all connected detour vertices of $G$. Hence it follows from Theorem 2.6 that $f c d n(G) \leq c d n(G)-|W|=b-(b-a)=a$. Now, since $c d n(G)=b$, it follows from Theorem 2.11 that any connected detour basis of $G$ is of the form $S=W \cup\left\{x_{1}, x_{2}, \ldots, x_{a}\right\}$, where $x_{i} \in\left\{u_{i}, v_{i}\right\}$ $(1 \leq i \leq a)$. Let $T$ be a subset of $S$ with $|T|<a$. Then there is a vertex $x_{j}(1 \leq j \leq a)$ such that $x_{j} \notin T$. Let $y_{j}$ be a vertex of $F_{j}$ distinct from $x_{j}$. Then $S^{\prime}=\left(S-\left\{x_{j}\right\}\right) \cup\left\{y_{j}\right\}$ is also a connected detour basis such that it contains $T$. Thus $S$ is not the unique connected detour basis containing 
$T$ and so $T$ is not a forcing set of $S$. Since this is true for all connected detour bases of $G$, it follows that $f c d n(G) \geq a$ and so $f c d n(G)=a$.

\title{
References
}

[1] F. Buckley and F. Harary, Distance in Graphs, Addison-Wesley, Reading MA, (1990).

[2] G. Chartrand, H. Escuadro and P. Zhang, Detour distance in graphs, J. Combin. Math. Combin. Comput., 53, pp. 75-94, (2005).

[3] G. Chartrand, L. Johns, and P. Zhang, Detour Number of a Graph, Util. Math. 64, pp. 97-113, (2003).

[4] G. Chartrand and P. Zhang, Distance in Graphs-Taking the Long View, AKCE J. Graphs.1. No.1, pp. 1-13, (2004).

[5] G. Chartrand and P. Zang, Introduction to Graph Theory, Tata McGraw-Hill, (2006).

[6] A. P. Santhakumaran and S. Athisayanathan, The connected detour number of a graph, J. Combin. Math. Combin. Comput., 69, pp. 205218, (2009).

\author{
A. P. Santhakumaran \\ Department of Mathematics \\ Hindustan University \\ Hindustan Institute of Technology and Science \\ Padur, Chennai-603 103, India \\ e-mail : apskumar1953@yahoo.co.in \\ and
}

\section{S. Athisayanathan}

Department of Mathematics

St. Xavier's College (Autonomous)

Palayamkottai - 627 002,

India

e-mail: athisayanathan@yahoo.co.in 